\title{
Selling cellular gels
}

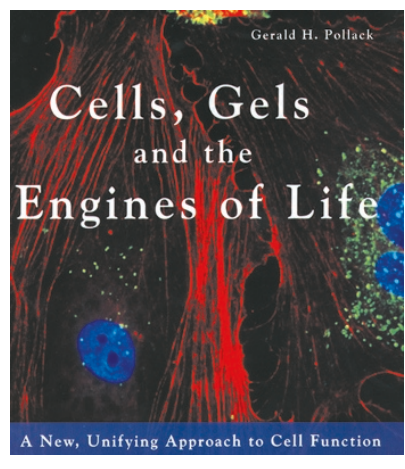

\author{
Cells, Gels and the Engines of Life
}

by G. H. Pollack

Ebner and Sons $•$ March 2001

Hardback $\$ 55$

Michael. W. Klymkowsky

A New, Unifying Approach to Cell Functio

here is no denying it, cells are dauntingly complex - densely packed with macromolecules of many sorts interacting in myriad ways. In the past, cellular complexity had been subsumed in the term 'protoplasm. The modern revolution in molecular biology has, in large measure, been based on the resolution of protoplasm into discrete structures - surface and endogenous membrane systems, membrane-delimited organelles (for example, mitochondria, chloroplasts and the nuclei of eukaryotic cells) and their embedded proteins, cytoskeletal filament systems and their associated proteins, chromosomes and the gene regulatory/replicative machinery, and a host of specialized macromolecular machines or 'somes' such as ribosomes, proteosomes, splicesomes and foldosomes (chaperones).

Modern cell-biology textbooks typically deal with these cellular components as distinct topics. Occasionally, they may include a few of David Goodsell's structurally realistic, but static drawings of cellular interiors, but they rarely deal with the dynamic realities of the living cell. It is a main thesis of Gerald Pollack's Cells, Gels and the Engines of Life that this molecular reductionism is altogether too naive and has missed something crucial - namely, the role of water generally and how its structuring determines cellular organization and function more specifically. There is some truth in this view - we are still in the early stages of placing the molecular machines of the cell into a functionally realistic context.

This is, however, not a carefully reasoned and objective presentation of the state of our knowledge and its limitations. It is highly polemical work whose admitted goal is a revolution in our understanding of cellular organization and function. Its style is folksy and 'us against them' to the point of being almost anti-scientific. In fact, its overall style is reminiscent of creationist writings. Pollack focuses observations that appear, at least superficially, to be inconsistent with what he paints as the current dogma of the cell-biology establishment. On the basis of these inconsistencies he discards - more or less lock, stock and barrel - an extremely large number of well-established observations in favour of a few scattered and almost anecdotal ones. Given that biology is built on the cross-checked results of many investigators, this can be a misleading strategy. Membrane channels and pumps are the first to go, but they are by no means the last. They are replaced with models that are based primarily on structured water.

That protoplasm has the qualities of a crowded, thixotropic colloidal solution is well appreciated (see E. B. Wilson's discussion of protoplasm in The Cell in Development and Heredity, Garland Publishing). That non-living colloids can display behaviours reminiscent of cells is also not in question. That modern textbooks generally ignore the fact that protoplasm behaves differently to a simple concentrated protein solution is also clear. That this implies that the view of the cell presented in the typical cell-biology textbook is fundamentally mistaken is, however, too broad a jump. More to the point, the author's approach does injury to the ideals of scientific inquiry.

Cells are about $70 \%$ water and packed with macromolecular components. How is cellular water organized? Previous studies estimated that roughly $90 \%$ of total cellular water is present as free, rather than bound or structured water. Nevertheless, there are arguments, which are reflected in Pollack's text (for example see http://www.consciousness.arizona.edu/hameroff/water2.html), that structured water is responsible for everything from membrane polarity to consciousness. Echoes of 'polywater' spring to mind.

What Pollack has done is collect a diverse set of observation that appear to fly in the face of the conventional view of cellular organization: these range from the apparent presence of channel-like electrical behaviour when microelectrodes are opposed to polymer surfaces and the maintenance of membrane potential when membranes are physi- cally breached, to the movement of singleheaded kinesin-type motor proteins. This type of scientific 'muck-making' has a certain appeal, as it points out gaps in our knowledge that are often glossed over in textbooks. $\mathrm{I}$, for one, have always been intrigued by the evidence that holes are routinely torn in the membranes of living cells by mechanical stresses.

Unfortunately, this is only the first step in developing a more sophisticated understanding of cellular processes. What Pollack fails to do is to bring an analytical and rigorous scepticism to these 'heretical' observations; moreover, he ignores much of what is already known. Consider his discussion of membrane channels: his basic premise is that their ability to conduct specific ions through a membrane is a "functionally insignificant" (page 21) by-product of their true role as "receptors". But the ramifications of this view are very poorly developed, and there is essentially no serious consideration given to the many structural and functional observations that support the channel/pump hypothesis. While pointing out that pure lipid bilayers are relatively impermeable to water, he dismisses as superfluous the recent characterization of water channels (aquaporins) as a solution to the observed permeability of biological membranes. Instead, he proposes that water leaks through membranes at the lipid/protein boundary layer. The data for water transport through aquaporins are never seriously considered.

There is a certain type of person who needs to turn the world upside down. As the boundaries of the mysterious disappear, this urge often grows stronger. As biology matures, it has become a study of the structure, regulation, function and interactions of molecular machines - and many of its mysteries have vanished. Pollack's book seeks to recover some of that mystery.

Michael Klymkowsky is in the Department of Molecular, Cellular and Developmental Biology, University of Colorado, Boulder, Colorado 80309-0347, USA.

e-mail:klym@spot.colorado.edu 